\title{
Optimizing Spectrum Sensing for Cognitive Radio Networks to Limit Interference on Primary User
}

\author{
Ramez Moh. Elaskary \\ Electronics and communications dept. \\ Institute of Aviation Engineering and Technology \\ Egypt
}

\begin{abstract}
Cognitive radio (CR) is an effective way to improve the utilization of spectrum resource. Spectrum sensing is playing an important role for finding free channels to be used by CR. One of the most critical issues in spectrum sensing is the interference on primary user (PU). In this work, a general optimal voting rule is studied to minimize the Bayes risk function in cooperative spectrum sensing. Furthermore, an algorithm to optimize the energy detection threshold for the CR users for any fusion rule is presented. On the other hand, an algorithm that determines the optimum fusion rule and optimum threshold that minimizes the false alarm probability while the missing probability is under constraint (bounded) is suggested.
\end{abstract}

\section{Keywords}

Cognitive radio, cooperative spectrum sensing, Bayes risk function, optimization, energy detector.

\section{INTRODUCTION}

Recent years, with the rapid development of wireless communication technology, more and more spectrum resources are needed to support the high data rate. Spectrum scarcity becomes a problem. The Federal Communications Commission (FCC) Spectrum Policy Task Force (SPTF) have demonstrated that the actual licensed spectrum is unoccupied most of the time [1]. Another work on spectrum occupancy measurements showed that the average spectrum occupancy from $30 \mathrm{MHz}$ to $3 \mathrm{GHz}$ over six cities is $5.2 \%$ [2]. The cognitive radio $\mathrm{CR}$ has been proposed [3], [4] to mitigate the conflict between spectrum scarcity and low spectrum efficiency.

One of the most challenging tasks in CR networks is spectrum sensing, which is required to opportunistically access the idle radio spectrum. Generally, the spectrum sensing techniques can be classified as energy detection, matched filter detection, and cyclostationary feature detection [5]. Among these techniques, energy detection has low complexity, low implementation cost, and demands no apriori knowledge about the primary user signal [6]. In this paper, the energy detection is considered as the local spectrum sensing scheme.

One of the great challenges of implementing spectrum sensing is the hidden terminal problem, this problem can be caused by many factors including severe multipath fading, shadowing, or inside buildings with high penetration loss, while a primary user (PU) is operating in the vicinity [7].

Due to the hidden terminal problem, a CR device causes unwanted interference to the PU (receiver) as the primary transmitters signal. The cooperative spectrum sensing can solve the hidden terminal problem in cognitive radio networks (CRNs). It has been shown that spectrum sensing performance

\author{
Yasmine Fahmy \\ Electronics and communications dept. \\ Faculty of engineering, Cairo University \\ Egypt
}

can be greatly improved with an increase of the number of cooperative partners [8].

In [9] the optimal number of SUs for the majority voting rule and the optimal detection threshold were derived to minimize the sum of false alarm probability and missed detection probability.

The rest of the paper is organized as follows. Section 2 presents the system model and defines the symbols that will be used through the paper. Section 3 presents the Bayes risk function and studies the optimal voting rule analytically and through simulations. Section 4 determines the optimum fusion rule and optimum threshold that minimizes the false alarm probability while the missing probability is bounded. Finally, section 5 concludes the paper.

\section{SYSTEM MODEL}

A CRN with K Secondary Users (SUs) and one central node are considered. Assume that each SU performs spectrum sensing independently and takes its local binary decision (the binary decision 0 or 1 which represent the channel is occupied or the channel is unoccupied respectively). The central node collects the $\mathrm{K}$ binary decisions from the K SUs to decide whether the PU is active or not.

The spectrum sensing can be modeled as a binary hypothesis testing problem with hypothesis $\mathrm{H}_{0}$ and $\mathrm{H}_{1}$ denoting the absence and presence of a PU, respectively. In the proposed model, the low-pass equivalent of the $\mathrm{i}^{\text {th }}$ sample of the received signal at the $\mathrm{k}^{\text {th }}$ radio is written as:

$$
r_{k}(i)= \begin{cases}w_{k}(i), & H_{0} \\ h_{k}(i) s(i)+w_{k}(i) & H_{1}\end{cases}
$$

Where $r_{k}(i)$ is the received signal at the $k^{\text {th }} \mathrm{CR}$ at the $i^{\text {th }}$ sample, $w_{k}(i)$ is the additive white Gaussian noise (AWGN) of the $k^{\text {th }} \mathrm{CR}$ at the $i^{\text {th }}$ sample, $h_{k}(i)$ is the complex gain of the sensing channel between the PU and the $k^{\text {th }} \mathrm{CR}$ at the $i^{\text {th }}$ sample, and $s(i)$ is the PU signal at the $i^{\text {th }}$ sample. Assume that the observation window of each radio has I samples, and sensing is performed with $\mathrm{K}$ radios. Then $i=1,2, \ldots . \mathrm{I}$ and $k=1,2, \ldots . . K$. Also assume sampling is done at the symbol rate, the sensing channel is time-invariant during the sensing process and error free.

For the $k^{\text {th }} \mathrm{CR}$ with the energy detector, the average probability of false alarm, the average probability of detection, and the average probability of missed detection over AWGN channels are given, respectively, by [10].

$$
\begin{aligned}
& P_{f, k}=\frac{\Gamma\left(u, \frac{\lambda_{k}}{2}\right)}{\Gamma(u)} \\
& P_{d, k}=Q_{u}\left(\sqrt{2 \gamma_{k}}, \sqrt{\lambda_{k}}\right)
\end{aligned}
$$


and

$P_{m, k}=1-P_{d, k}$

Where $\gamma_{k}$ and $\lambda_{k}$ denote the instantaneous signal-to-noise ratio $(S N R)$ and the energy detection threshold of the $k^{\text {th }} \mathrm{CR}$ respectively, $u$ is time-bandwidth product of the energy detector, $\Gamma(a, x)$ is the incomplete gamma function, $\Gamma(a)$ is the gamma function, and $Q_{u}(a, b)$ is the generalized Marcum Q-function.

In cooperative spectrum sensing each $\mathrm{CR}$ device make a binary decision depending on its local observation and then forwards one bit of the decision $D_{k}$ (1 standing for the presence of the PU, 0 for the absence of the PU) to the central node through an error-free channel. At the central node, all 1bit decisions are fused together according to the following logic rule

$Z=\left\{\begin{array}{lll}\sum_{k=1}^{K} D_{k} \geq n & , \quad H_{1} \\ \sum_{k=1}^{K} D_{k}<n & , \quad H_{0}\end{array}\right.$

Where the threshold $n$ is an integer, representing the " $n$-outof-K" voting rule.

Assume the distance between the PU and any CR device is very large comparing with the distance between any two CRs, then the received signal at all the CRs have almost identical path losses. Therefore, in the case of an AWGN environment, it can assume that $\gamma_{1}=\cdots=\gamma_{\mathrm{K}}=\gamma$. These assumptions result in $P_{f, k}$ being independent of $\mathrm{k}$, then it can be denoted as $P_{f}$. In the case of an AWGN channel, $P_{d, k}$ is independent of $\mathrm{k}$ $\left(P_{d}\right)$.

In the case of a Rayleigh fading environment, it is reasonable to assume there are have independent and identically distributed (i.i.d.) Rayleigh fading with the instantaneous SNRs $\gamma_{1}, \ldots \ldots, \gamma_{\mathrm{K}}$ being i.i.d. exponentially distributed random variables with the same mean $\gamma$. Furthermore, assume that all CRs use the same threshold $\lambda$. In the case of fading channel, let $P_{d}$ be $P_{d, k}$ averaged over the statistics of $\gamma_{k}$. Where $P_{d}=\int_{\gamma} Q_{u}\left(\sqrt{2 \gamma_{k}}, \sqrt{\lambda_{k}}\right) f_{\gamma}(x) d x$

In a Rayleigh fading channel $P_{d}$ is given by [11] as

$\mathrm{P}_{\mathrm{d}}^{\mathrm{Ray}}=\frac{\Gamma\left(\mathrm{u}-1, \frac{\lambda_{\mathrm{k}}}{2}\right)}{(\mathrm{u}-2) !}+\left(\frac{1+\overline{\gamma_{\mathrm{k}}}}{\overline{\bar{\gamma}_{\mathrm{k}}}}\right)^{\mathrm{u}-1} \exp \left(\frac{-\lambda_{\mathrm{k}}}{2\left(1+\overline{\gamma_{\mathrm{k}}}\right)}\right)\left[1-\frac{\Gamma\left(\mathrm{u}-1, \frac{\overline{\gamma_{\mathrm{k}}} \lambda_{\mathrm{k}}}{2\left(1+\overline{\gamma_{\mathrm{k}}}\right)}\right)}{(\mathrm{u}-2) !}\right]$

Where $\overline{\gamma_{k}}$ is the average $S N R$ of the $k^{\text {th }}$ CR user. Moreover $P_{f, k}$ is independent on instantaneous $S N R \gamma_{k}$, and $P_{f, k}$ is given by (1).

Therefore, the false alarm probability of cooperative spectrum sensing is given by

$Q_{f}=\operatorname{Prob}\left\{H_{1} \mid H_{0}\right\}=\sum_{l=n}^{K}\left(\begin{array}{l}K \\ l\end{array}\right) P_{f}^{l}\left(1-P_{f}\right)^{K-l}$

And the missed detection probability of cooperative spectrum sensing is given by

$Q_{m}=\operatorname{Prob}\left\{H_{0} \mid H_{1}\right\}=1-\sum_{l=n}^{K}\left(\begin{array}{l}K \\ l\end{array}\right) P_{d}^{l}\left(1-P_{d}\right)^{K-l}$

\section{BAYES RISK FUNCTION OPTIMIZATION}

\subsection{Optimum Fusion Rule with Minimum Risk function}

In [9] the author defined, the total error as equal $Q_{f}+Q_{m}$ (i.e. he gave $Q_{f}$ and $Q_{m}$ equal weights), then he got an expression for the optimal value of $n$ for the " $n$-out-of- $K$ " rule.

In this work, the more general case where $Q_{f}$ and $Q_{m}$ have unequal weights is considered because of in $\mathrm{CR}$ the missing probability $Q_{m}$ causes a harmful interference on the PU, while
$Q_{f}$ decreases the spectrum utilization. So, it assume that $Q_{m}$ is more "dangerous" than $Q_{f}$. Therefore, an expression for the optimal voting rule that minimizes the Bayes risk function in case of unequal weights for $Q_{m}$ and $Q_{f}$ is needed to derive.

Let's now, define the Bayes risk function $\mathcal{R}$ as:

$\mathcal{R}=c_{00} P_{00} P_{0}+c_{11} P_{11} P_{1}+c_{10} P_{10} P_{0}+c_{01} P_{01} P_{1}$

(8)

Where,

- $\quad c_{i j}$ is the cost of deciding the channel is $H_{i}$ while the channel is $H_{j}$.

- $P_{i j}$ is the probability of deciding the channel is $H_{i}$ while the channel is $H_{j}$,

- $P_{i}$ is the probability that the channel is $H_{i}$

In this case, it can assume that $c_{00}=c_{11}=0$ (i.e., the correct decision costs are zero), $P_{10}=Q_{f}$, and $P_{01}=Q_{m}$ then equation (8) becomes:

$\mathcal{R}=c_{10} P_{0} Q_{f}+c_{01} P_{1} Q_{m}$

Without loss of generality assume $\mathcal{R}$ is normalized such that, $c_{10} P_{0}+c_{01} P_{1}=1$

Then it can be rewritten as

$\mathcal{R}=\beta Q_{f}+(1-\beta) Q_{m}$

Where $\beta=c_{10} P_{0}$, which represents the weight of $Q_{f}$ and $(1-\beta)$ represents the weight of $Q_{m}$. So that, the normalized risk cost function $\mathcal{R}$ represents the error rate probability.

Therefore the normalized cost function $\mathcal{R}$ is a function of $n$ (voting rule), $\lambda$ (energy detection threshold), $\mathrm{K}$ (total number of cooperated secondary users), and $\beta$ (weight of false alarm probability). It should be noted that there is no optimum value for $\mathrm{K}$ that minimizes the normalized cost function $\mathcal{R}$, as $\mathrm{K}$ increases the cost function decreases. Through this paper, studying the optimum voting rule $n_{o p}$ is concentrated, and the optimum energy detection threshold $\lambda_{o p}$ that minimize the cost function $\mathcal{R}$ for different weights $\beta$, and constant $\mathrm{K}$.

By substituting (6) and (7) in (10), the normalized cost function $\mathcal{R}$ can rewrite as

$\mathcal{R}(n)=\sum_{l=n}^{\mathrm{K}}\left(\begin{array}{l}\mathrm{K} \\ l\end{array}\right)\left[\begin{array}{c}{\left[\mathrm{P}_{\mathrm{f}}^{\mathrm{l}}\left(1-\mathrm{P}_{\mathrm{f}}\right)^{\mathrm{K}-\mathrm{l}}-(1-\beta)\left(1-\mathrm{P}_{\mathrm{m}}\right)^{\mathrm{l}} \mathrm{P}_{\mathrm{m}}{ }^{\mathrm{K}-\mathrm{l}}\right]} \\ -1\end{array}\right.$

To get $n$ that minimizes $\mathcal{R}$, differentiate $\mathcal{R}$ with respect to $n$ and equating it to zero:

$\frac{\partial \mathcal{R}}{\partial n} \cong[\mathcal{R}(n+1)-\mathcal{R}(n)]=0$

By subtracting $\mathcal{R}(n)$ from $\mathcal{R}(n+1)$,

$\beta P_{f}^{n_{o p}}\left(1-P_{f}\right)^{K-n_{o p}}-(1-\beta)\left(1-P_{m}\right)^{n_{o p}}\left(P_{m}\right)^{K-n_{o p}}=0$

After some mathematical manipulations, it get the following as a value for $n_{o p}$

$n_{o p}=\frac{K \ln \left(\frac{P_{m}}{1-P_{f}}\right)-\ln \left(\frac{\beta}{1-\beta}\right)}{\left(\ln \left(\frac{P_{f}}{1-P_{m}}\right)+\ln \left(\frac{P_{m}}{1-P_{f}}\right)\right)}$

$n_{o p}$ Should be an integer value and always less than or equal to the number of SUs, then $n_{o p}$ can be rewritten as, 
$n_{o p}=\min \left(K,\left\lceil\frac{K \ln \left(\frac{P_{m}}{1-P_{f}}\right)-\ln \left(\frac{\beta}{1-\beta}\right)}{\ln \left(\frac{P_{f}}{1-P_{m}}\right)+\ln \left(\frac{P_{m}}{1-P_{f}}\right)}\right\rceil\right)$

This is the optimal value of $n$ (for the fusion rule " $n$-out-of$K$ ") in case of $Q_{f}$ and $Q_{m}$ have unequal weights. Then $n_{o p}$ depends on the total number of users $\mathrm{K}$, the energy detection threshold $\lambda$, the weight $\beta$, and the $S N R$. The behavior of $n_{o p}$ with these parameters is illustrated in Fig. 1 where $n_{o p}$ is plotted versus $\mathrm{K}$ at constant $\lambda$ in Rayleigh fading channel for different $S N R$ and different $\beta$.

Fig. 2 illustrates the $n_{o p}$ dependency on $\lambda$, for each value of $\lambda$ there is a different value for $n_{o p}$ at $\mathrm{K}=15$ for different $\beta$. The figure shows that as $\lambda$ increases $n_{o p}$ decreases and also when $\beta$ decreases, $n_{o p}$ decreases.
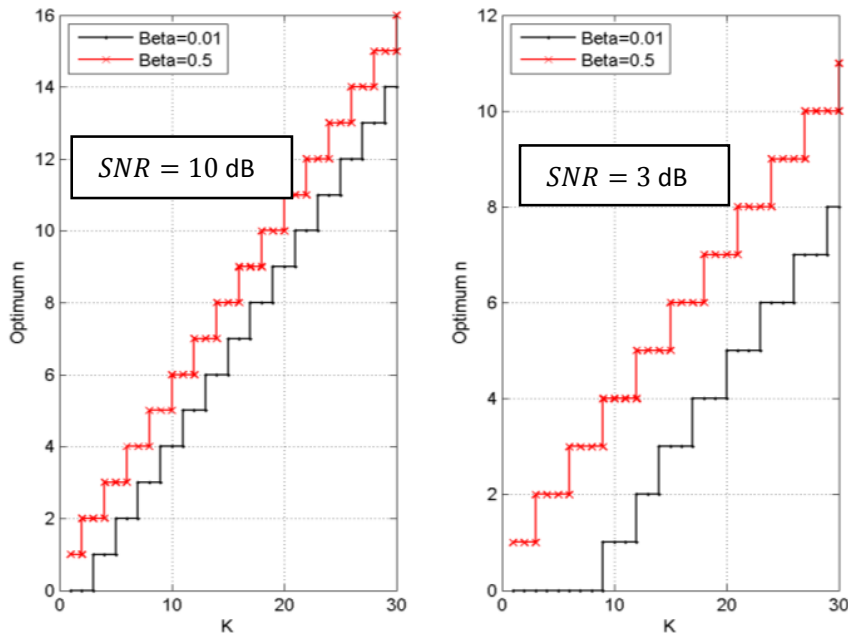

Fig. $1 n_{o p}$ versus number of users K of cooperative spectrum sensing in Rayleigh fading channel for different $S N R$, at constant energy detection threshold $\lambda=8$ for varies values of $\beta=\{0.01,0.5\}$.

In Fig. $3, Q_{m}, Q_{f}$, and $\mathcal{R}$ are plotted versus different $\mathrm{K}$ at $\lambda=8$ and $S N R=10 \mathrm{~dB}$, from the figure at $\beta=0.5, Q_{m}$ is almost equal to $Q_{f}$, and as $\beta$ decreases $Q_{m}$ decreases and $Q_{f}$ is increases. The improvement gain between cooperative system $(K>1)$ and none cooperative $(K=1)$ can be noted.

The optimum value $n_{o p}$ in equation (11) can be rewritten as

$n_{o p}=\frac{K \ln \left(\frac{P_{m}}{1-P_{f}}\right)}{\left(\ln \left(\frac{P_{f}}{1-P_{m}}\right)+\ln \left(\frac{P_{m}}{1-P_{f}}\right)\right)}-\frac{\ln \left(\frac{\beta}{1-\beta}\right)}{\left(\ln \left(\frac{P_{f}}{1-P_{m}}\right)+\ln \left(\frac{P_{m}}{1-P_{f}}\right)\right)}$

Where the first term in (13) represents $n_{o p}$ when the weight $\beta$ is neglected (i.e. $\beta=0.5$ ) as in [9] or

$n_{o p}=\frac{K \ln \left(\frac{P_{m}}{1-P_{f}}\right)}{\left(\ln \left(\frac{P_{f}}{1-P_{m}}\right)+\ln \left(\frac{P_{m}}{1-P_{f}}\right)\right)}$

Fig. 4 illustrates that the normalized risk $\mathcal{R}$ of (10) when $n_{o p}$ is calculated using (13) and using (14). In the figure, it is clear that the performance is degraded if the effect of $\beta$ is neglected.
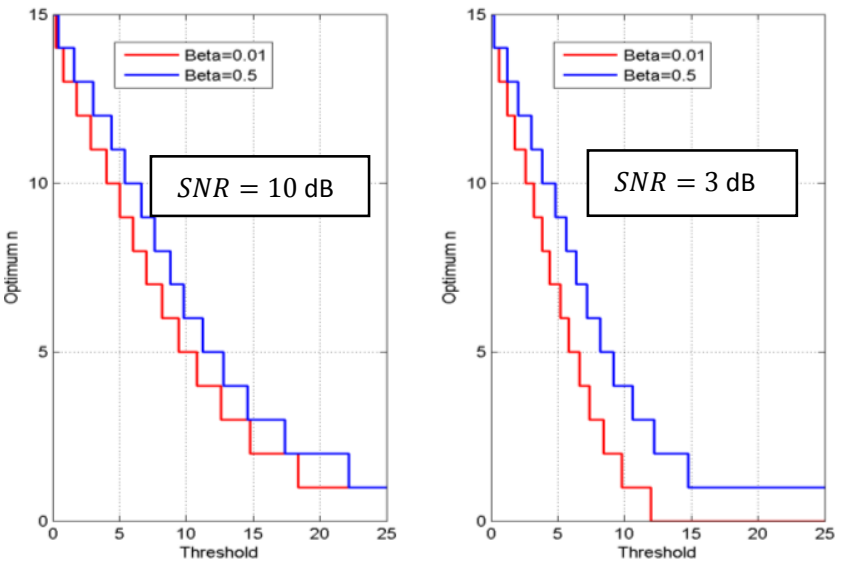

Fig. 2 Optimum fusion rule $\boldsymbol{n}_{o p}$ versus detection threshold $\lambda$ of cooperative spectrum sensing at $K=15$ in Rayleigh fading channel for different $S N R$, for varies values of $\boldsymbol{\beta}=\{0.01,0.5\}$.
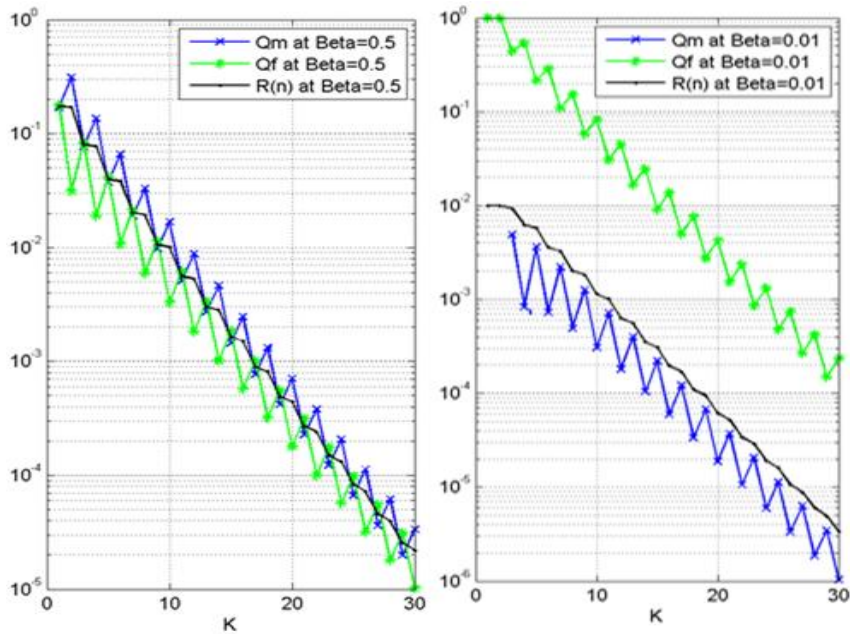

Fig. $3 Q_{m}, Q_{f}$, and $\mathcal{R}$ versus $\mathrm{K}$ in Rayleigh fading channel at $S N R=10 \mathrm{~dB}$ and $\lambda=8$ for varies values of $\beta=$ $\{0.5,0.01\}$.
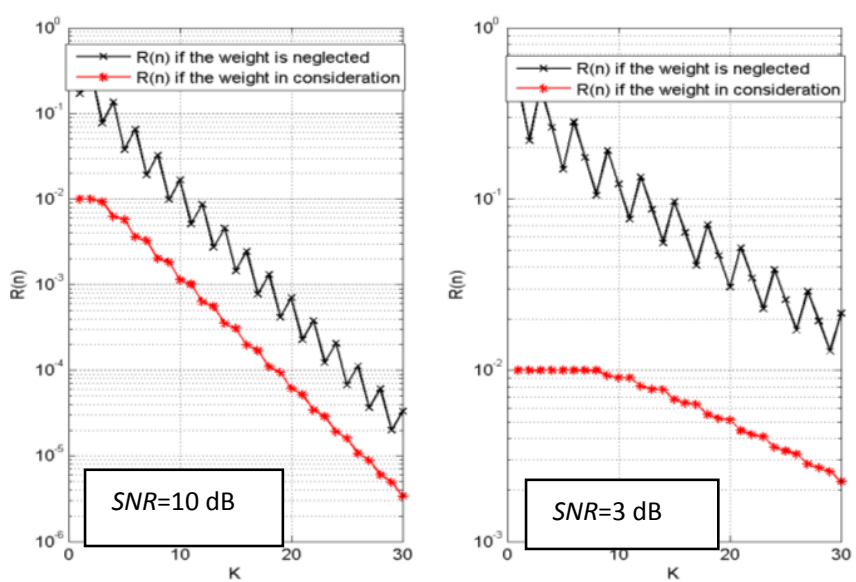

Fig. $4 \mathcal{R}(n)$ versus $K$ for $\beta=0.01$ if $\beta$ is neglected and if $\beta$ in consideration in Rayleigh fading channel for different $S N R$, and $\lambda=8$. 


\subsection{Optimal Energy Detection Threshold}

If $\mathrm{K}$ and $S N R \quad \gamma$ are known and $n_{o p}$ is expressed as in (12) then, to determine the optimum energy detection threshold $\lambda_{\text {op }}$ that minimizes $\mathcal{R}(n)$ such that

$$
\lambda_{\text {op }}=\min _{\lambda}[\mathcal{R}(n)]=\min _{\lambda}\left(\beta Q_{f}+(1-\beta) Q_{m}\right)
$$

The total error rate in terms of $\lambda$ has a global minimum in $\lambda$ for each $n$ [9]. This means that there exist only one value of $\lambda$ that minimizes $\mathcal{R}(n)$.

Then, to get an expression for $\lambda_{o p}$,differentiate $\mathcal{R}$ with respect to $\lambda$ and equating it to zero.

$\beta \frac{\partial Q_{f}}{\partial \lambda}+(1-\beta) \frac{\partial Q_{m}}{\partial \lambda}=0$

The expressions for $\frac{\partial Q_{m}}{\partial \lambda}$ and $\frac{\partial Q_{f}}{\partial \lambda}$ were derived in [9], however, it is quite difficult to get a closed-form solution for $\lambda$. Because there exists one solution, it can employ any one dimensional optimization algorithms (e.g., Bisection search, Fibonacci search, Golden section search, Newton search) to find $\lambda_{o p}$. An example to find $\lambda_{o p}$ is given in algorithm 1 .

\section{Algorithm 1}

Find $\lambda_{o p}$ assume $\mathrm{K}, n$, and $S N R \gamma$ are known

$$
\begin{aligned}
& \text { Input } \mathrm{K}, n, S N R \gamma \text {, and } \Delta(\Delta \text { is the tolerance of accuracy of } \\
& \left.\lambda_{o p}\right) \text {. } \\
& \text { Initialize } \lambda^{(-1)}=0, \lambda^{(0)}=1, l=0 \\
& \text { while } \lambda^{(l)}-\lambda^{(l-1)}>\Delta \\
& \text { calculate } \lambda^{(l+1)} ; \lambda^{(l+1)}=\frac{\frac{\partial \mathcal{R}\left(\lambda^{(l)}\right)}{\partial \lambda} \lambda^{(l-1)}-\frac{\partial \mathcal{R}\left(\lambda^{(l-1)}\right)}{\partial \lambda} \lambda^{(l)}}{\frac{\partial \mathcal{R}\left(\lambda^{(l)}\right)}{\partial \lambda}-\frac{\partial \mathcal{R}\left(\lambda^{(l-1)}\right)}{\partial \lambda}} \\
& l=l+1 \\
& \text { end while } \\
& \text { Output } \lambda_{o p}=\lambda^{(l)}
\end{aligned}
$$

\subsection{Optimum Fusion Rule and Energy Threshold}

In this subsection the objective is determination of $\left(\lambda_{o p}, n_{o p}\right)$ that yields the lowest error rate probability (minimum $\mathcal{R}$ ). This process is proposed in algorithm 2 [12].

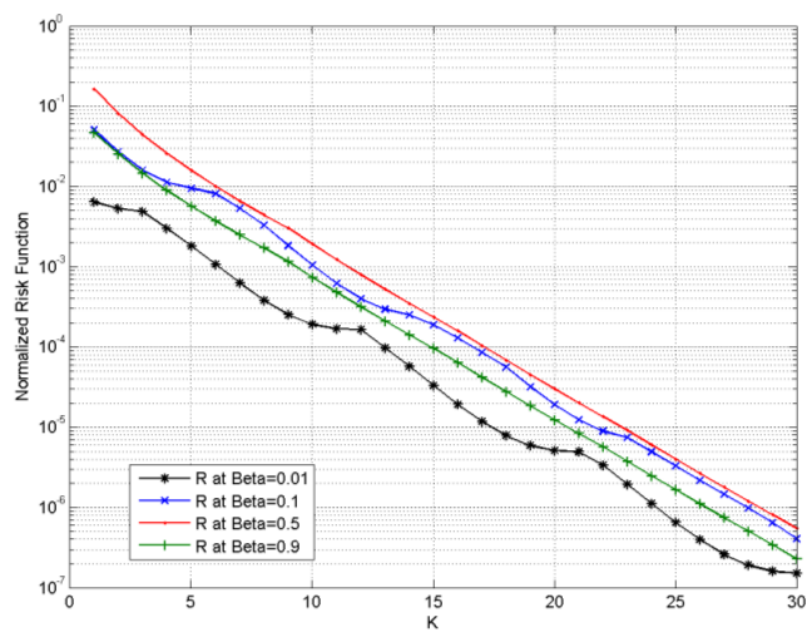

Fig. $5 \mathcal{R}\left(n_{o p}, \lambda_{o p}\right)$ versus $K$ in Rayleigh fading channel with $S N R=10 \mathrm{~dB}$ and for $\beta=\{0.01,0.1,0.5,0.9\}$.

Fig. 5 illustrates $\mathcal{R}\left(n_{o p}, \lambda_{o p}\right)$ versus $\mathrm{K}$ for different values of $\beta$, from the figure, the improvement on $\mathcal{R}\left(n_{o p}, \lambda_{o p}\right)$ can be noted comparing with the $\mathcal{R}\left(n_{o p}\right)$ of Fig. 3 which is evaluated for $n_{o p}$ and a fixed value of $\lambda$.

Fig. 6 illustrates the optimum voting rule $n_{o p t}$ versus the number of users ' $\mathrm{K}$ ' of cooperative spectrum sensing in Rayleigh fading channel with $S N R=10 \mathrm{~dB}$ for $\beta=$ $\{0.01,0.5\}$. From the figure there is a very large difference between $n_{o p}$ at $\beta=0.01$ and at $\beta=0.5$ can be noted. If the weight $\beta$ is neglected, degradation in the performance of the system will occur.

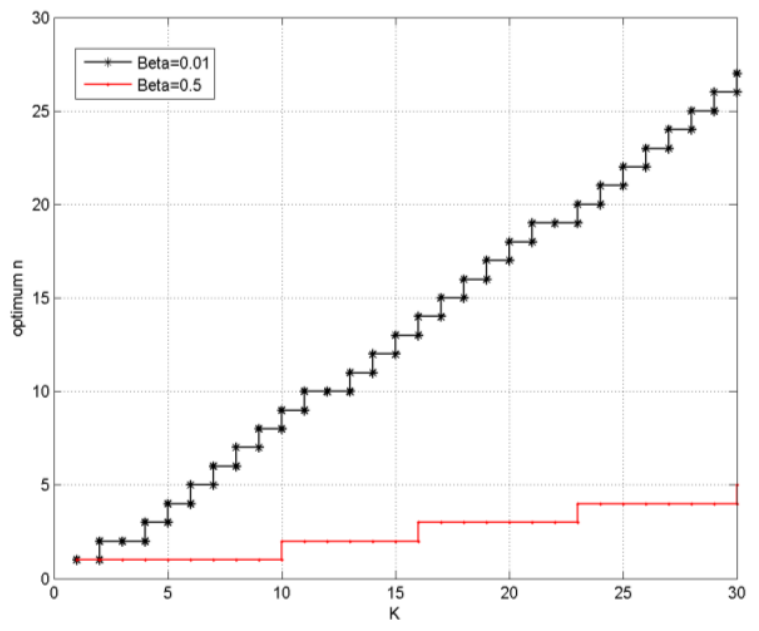

Fig. $6 n_{o p}$ versus $K$ in Rayleigh fading channel with $S N R=10 \mathrm{~dB}$ and for $\beta=\{0.01,0.5\}$.

\section{OBJECTIVE-CONSTRAINT OPTIMIZATION}

So far, the case of unequal weights for the missing and false alarm probabilities is considered. In this section, a technique that optimize $n$ and $\lambda$, which denoted as $n_{o p}$ and $\lambda_{o p}$ that minimizes the false alarm probability $Q_{f}$ with constraint on $Q_{m}$ and considering a fixed value of K. Then this problem is an optimization problem with constraint.

$F=Q_{f}+\Psi\left(Q_{m}-\varepsilon\right)$

(17)

Where $Q_{f}$ is given as in (6), $Q_{m} \leq \mathcal{E}$ is the constraint and $\Psi$ is the Lagrangian multiplier.

$\frac{\left(\frac{\partial Q_{f}}{\partial n}\right)}{\left(\frac{\partial Q_{m}}{\partial n}\right)}=\frac{\left(\frac{\partial Q_{f}}{\partial \lambda}\right)}{\left(\frac{\partial Q_{m}}{\partial \lambda}\right)}=\Psi$

This optimization problem is solved to get $n_{o p}$ and $\lambda_{o p}$. It is quite difficult to get the closed-form solution of $n_{o p}$ and $\lambda_{o p}$, therefore, an algorithm is proposed to determine them. 


\section{Algorithm 2}

Find $\lambda$ and $n$ to get minimum $Q_{f}$ such that $Q_{m} \leq \mathcal{E}$ assume $\mathrm{K}$ and $S N R \gamma$ are known.

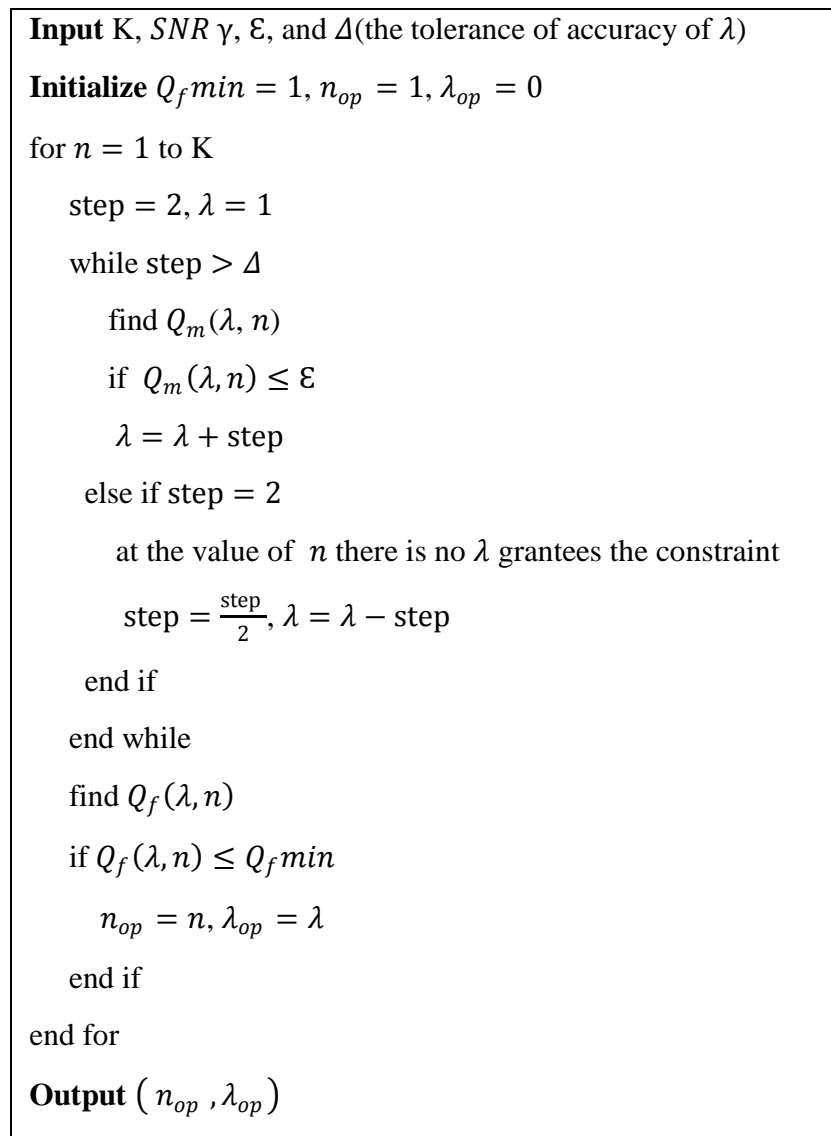

Fig. 7 illustrates $\lambda_{o p}$ versus $n$ when using algorithm 2 at $\varepsilon=10^{-4}, \mathrm{~K}=15$, and $S N R=10 \mathrm{~dB}$. Fig. 8 illustrates $Q_{m}, Q_{f}$ and $\mathcal{R}$ versus n, in the figure $Q_{m} \leq 10^{-4}, n_{o p}=2$ the value that minimizes $Q_{f}$ and the optimum energy detection threshold $\lambda_{o p}=15$ (from Fig. 7).

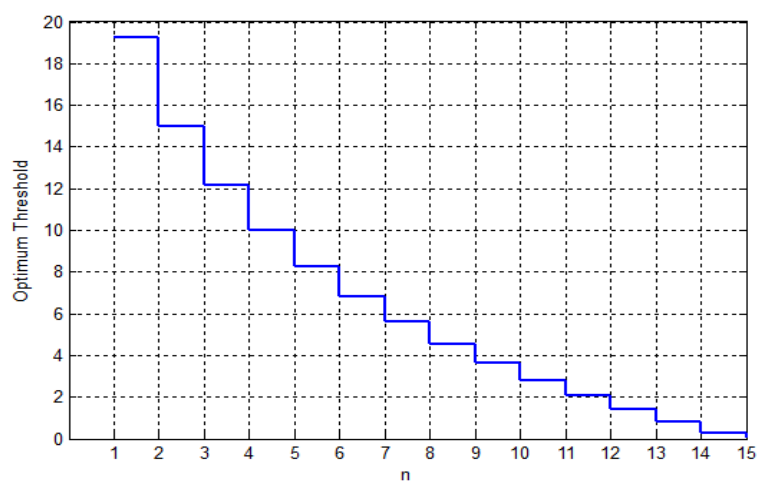

Fig. $7 \lambda_{o p}$ versus $n$ at $K=15, Q_{m} \leq 10^{-4}$ in Rayleigh fading channel with $S N R=10 \mathrm{~dB}$.

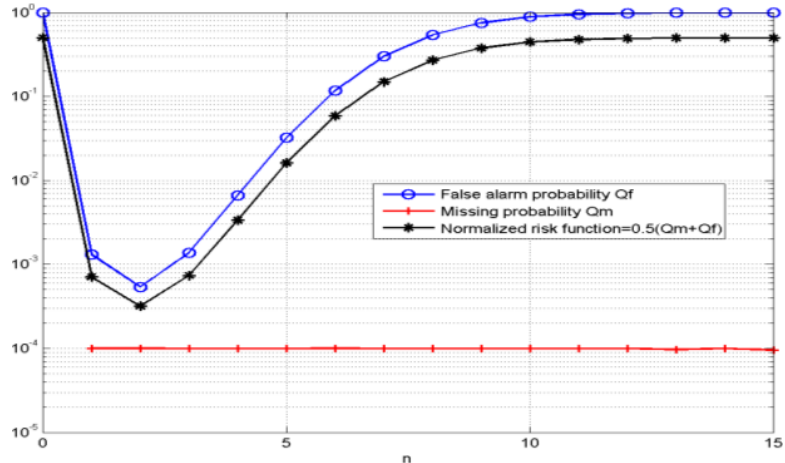

Fig. $8 Q_{m}, Q_{f}$, and $\mathcal{R}$ versus $n$ at $K=15, Q_{m} \leq 10^{-4}$ in Rayleigh fading channel with $S N R=10 \mathrm{~dB}$.

\section{CONCLUSION}

In this paper the two parameters: the optimal fusion rule and the optimum threshold to minimize the interference on the PU are studied. An unequal weight Bayes risk function is defined, derive an expression to determine the optimum fusion rule, and an algorithm to evaluate the optimum threshold is proposed. In addition, an optimal spectrum sensing problem was formulated by optimizing both fusion rule and threshold. Another problem where the missing probability is guarantee to be less than a certain threshold and minimizes the false alarm probability is proposed, the optimum fusion rule and the optimum threshold is evaluated for this problem.

\section{ACKNOWLEDGMENTS}

I appreciate the help offered by the journal staff. As they give the means and the spirit to realize a good work.

I would like to thank my father may Allah have mercy on him so much and I wish I could be present with me now.

Many thanks go to my mother and my wife for their continuous support and encouragement during all working daysandnights.

\section{REFERENCES}

[1] Federal Communications Commission, "Spectrum Policy Task Force,” Rep. ET Docket no. 02-135, Nov. 2002.

[2] M. A. McHenry, "NSF spectrum occupancy measurements project summary," Shared Spectrum Company Report, Aug. 2005. [Online] Available: http://www.sharedspectrum.com.

[3] J. Mitola and G. Q. Maguire, "Cognitive radio: making software radios more personal," IEEE Pers. Commun., vol. 6, pp-18, Aug. 1999.

[4] S. Haykin, "Cognitive radio: brain-empowered wireless communications," IEEE J. Select. Areas Commun., vol. 23, pp. 201-220, Feb. 2005.

[5] I. Akyildiz, W. Lee, M. Vuran, and S. Mohanty, "NeXt generation/ dynamic spectrum access/cognitive radio wireless networks: a survey," Computer Networks 50 (13) (2006) 2127-2159.

[6] R. Tandra and A. Sahai, "SNR walls for signal detection," IEEE J. Select. Topics in Signal Processing, vol. 2, no. 1, pp. $4-17$, Feb. 2008.

[7] D. Cabric, S. M. Mishra, and R. W. Brodersen, "Implementation issues in spectrum sensing for cognitive radios," in Proc. Asilomar Conf. Signals, Systems, Computers, Nov. 2004, vol. 1, pp. 772-776. 
[8] K. B. Letaief and W. Zhang, "Cooperative communications for cognitive radio," Proc. IEEE, vol. 97, no. 5, pp. 878-893, May 2009.

[9] W. Zhang, R. Mallik, and K. Letaief, "optimization of cooperative spectrum sensing with energy detection in cognitive radio networks," IEEE Trans. Wireless Commun., vol. 8, pp. 5761-5766, Dec. 2009.

[10] F. F. Digham, M.-S. Alouini, and M. K. Simon, "On the energy detection of unknown signals over fading channels," in Conf. Rec. IEEE Int. Conf. Commun.
(ICC'03), Anchorage, AK, USA, May 2003, pp. 3575 3579.

[11] F. F. Digham, M.-S. Alouini, and M. K. Simon, "On the energy detection of unknown signals over fading channels," IEEE Transactions on Communications, vol. 55, no. 1, pp. 21-24, Jan. 2007.

[12] H.Nguyen,T.Duy,H.Kong, “An optimal cooperative spectrum sensing method in cognitive radio network over Rayleigh fading channel. 